zeigen, kann man mit einiger Sicherheit extrapolieren. Mit abnehmendem Mengenverhältnis, d. h. mit abnehmender Kaltluftmenge nimmt die Erhitzung $a b$, jedoch wächst die Unterkühlung und zwar so, daß die Temperaturdifferenz zwischen Heiß- und Kaltluft bis zum Verhältnis $\gamma=0,3$ herunter, kaum abnimmt. Die größte Unterkühlung tritt bei $\gamma=0,3$ mit einer Kaltlufttemperatur von

$7^{\circ} \mathrm{C}$ auf. Mit weiter abnehmendem Mengenverhältnis nähern sich die Heiß- und die Kaltluft. temperaturen der Eintrittstemperatur und fallen bei $\gamma=0$ mit dieser zusammen, so daß also in diesem Grenzfall keine Trennwirkung besteht. Dies ist leicht $\mathrm{zu}$ verstehen, denn wenn kein Kaltluftstrahl austritt, so kann auch keine Luft im Rotationsfeld gekühlt werden und bei kleinen $\gamma$-Werten ist die Relativgeschwindigkeit der zu kühlenden Luft gegenüber der rotierenden zu gering, um genügend Turbulenz entstehen zu lassen.

\title{
Die Dissoziation des Schwefeldampfes II
}

\author{
Von H. Braune und E. Steinbacher
}

Aus dem Institut für physikalische Chemie der Technischen Hochschule Hannover

\author{
(Z. Naturforschg. 7 a, 486-493 [1952]; eingegangen am 28. März 1952)
}

\begin{abstract}
Bei der spektralphotometrischen Untersuchung der im sichtbaren Teil des Spektrums liegenden Absorptionsbanden des Schwefeldampfes wurde ein Kontinuum gefunden, das sein Maximum bei $5100 \AA$ hat. Seine Intensität bei dieser Wellenlänge wurde unter systematischer Variation von Druck und Temperatur mit der Druck- und Temperaturabhängigkeit der molaren Konzentrationen der verschiedenen im Schwefeldampf vorkommenden Molekülarten verglichen. Es ergab sich, daß die Intensität gut parallel geht mit der nach Braune, Peter und Neveling berechneten Konzentration des $\mathbf{S}_{4}$-Moleküls. Damit ist erwiesen, daß die von Preun er und $\mathbf{S c h}$ u p p angenommenen Molekülarten $\mathbf{S}_{8}, \mathbf{S}_{6}$ und $\mathbf{S}_{2}$ nicht ausreichen, um den Dampfzustand des Schwefels zu beschreiben, die Molekülart $\mathbf{S}_{4}$ tritt ebenfalls in meßbaren Konzentrationen auf. Das diffuse Bandensystem im kurzwelligen Teil des sichtbaren Spektrums kann nach dem Ergebnis dieser Arbeit nicht dem $\mathbf{S}_{2}$ zugeordnet werden, sondern dürfte gleichfalls dem $\mathbf{S}_{4}$-Molekül zugehören.
\end{abstract}

$\mathrm{D}$ ie Diskussion der in unserem Institut durchgeführten Messungen ${ }^{1}$ der Dichte des Schwefeldampfes von 350 bis $1000^{\circ} \mathrm{C}$ hatte zu dem Ergebnis geführt, daß entgegen der von Preuner und Schupp ${ }^{2}$ gemachten Annahme, wonach im SDampf nur $\mathrm{S}_{8}, \mathrm{~S}_{6}$ und $\mathrm{S}_{2}$ vorhanden sein sollten, auch die Existenz von $\mathrm{S}_{4}$ angenommen werden mußte. Die zur Darstellung des gesamten Versuchsmaterials geeigneten Dissoziationsgleichungen waren:

$$
\begin{aligned}
& \log K_{4}=\log \frac{p_{2}{ }^{2}}{p_{4}}=-\frac{28400}{4,57 T}+9,85, \\
& \log K_{6}=\log \frac{p_{2}{ }^{3}}{p_{6}}=-\frac{63710}{4,57 T}+20,97, \\
& \log K_{8}=\log \frac{p_{2}{ }^{4}}{p_{8}}=-\frac{92180}{4,57 T}+30,76 .
\end{aligned}
$$

Da sich nun mit fortschreitender Dissoziation bekanntlich auch die Farbe des S-Dampfes ändert,

${ }^{1}$ H. Braune, S. Peter u. V. Neveling, Z. Naturforschg. 6a. $32\lceil 1951\rceil$.

2 G. Preuner u. W. Schupp. Z. physik. Chem. Bs. 1.29 | 19091. lag es nahe, zu untersuchen, ob die Anwesenheit von $\mathrm{S}_{4}$ sich durch Messung der optischen Absorption im sichtbaren Gebiet bestätigen läßt. Schon Rosen und Mitarbeiter ${ }^{3}$ sowie D'Or ${ }^{4}$ haben das Absorptionsspektrum des S-Dampfes bei variierter Temperatur und Dichte photographiert. Von beiden Autoren wurde darauf hingewiesen, daß ihr qualitativer Befund nur schwer mit den von Preuner und Schupp angegebenen Daten in Einklang zu bringen sei.Wir haben nun mit Hilfe eines Spektralphotometers quantitative Messungen der Absorption im sichtbaren Gebiet ausgeführt, über deren Ergebnis im folgenden berichtet wird.

$$
\text { Experimenteller Teil }
$$

Die Temperaturen lagen bei diesen Versuchen zwischen 300 und $940^{\circ} \mathrm{C}$, die Gesamtkonzentrationen zwischen 43,5 und $1060 \mathrm{mg} / \mathrm{l}$.

${ }^{3}$ B. Rosen u. L. Neven, C. R. hebd. Séances Acad. Sci. 203, 663 [1936]. B. Rosen u. N. Morguleff, C. R. hebd. Séances Acad. Sci. 208, 273 [1939].

${ }^{4}$ L. I'Or. ('. R. hebd. Séances Acad. Sci. 201. 1026 | $1935 \mid$. 
Das Photometer nach König und Martens'war so eingerichtet, daß der Vergleichsștrahl mit Hilfe von Ablenkprismen um den Ofen mit den Absorptionsrohren herumgeführt wurde. Der Ofen, der in der optischen Achse des Hauptstrahlenganges aufgestellt wurde, hatte eine Länge von $54 \mathrm{~cm}$ und eine Bohrung von $6 \mathrm{~cm}$ Durchmesser; er bestand aus $V_{2} A-S t a h l$ von $2 \mathrm{~cm}$ Wandstärke und war mit einer Hauptwicklung und 2 Hilfswicklungen an beiden Enden versehen. Durch Abgleichung der Stromstärken konnte Temperaturkonstanz längs der Absorptionsrohre erzielt werden. Im ungünstigsten Falle, bei Verwendung des längsten Absorptionsrohres von $47 \mathrm{~cm}$ betrug die Temperaturdifferenz zwischen Mitte und Ende des Rohres $3^{0} \mathrm{C}$, bei den kürzeren Rohren war sie unmerklich. Als Absorptionsrohre dienten Rohre aus Quarzglas mit aufgeschmolzenen planparallelen Platten von $3 \mathrm{~cm}$ Durchmesser und 1,5, 3, 24 und $47 \mathrm{~cm}$ Länge.

Zur Vermeidung von Änderungen der Justierung des Absorptionsrohres infolge der Wärmeausdehnung des Ofens wurde es jeweils an ein langes Quarzrohr angeschmolzen, das auf beiden Seiten außerhalb des Ofens fest gelagert wurde, so daß das Absorptionsrohr die Ofenwand nicht berührte. Das Quarzrohr diente gleichzeitig zur Aufnahme des Pt-PtRh-Thermoelementes, dessen Thermokraft mit einem Millivoltmeter gemessen wurde. Das Element war mit den Schmelzpunkten von $\mathrm{Zn}$, Cd und Au geeicht. Die Fehler der Temperaturangaben dürften $2-3^{\circ} \mathrm{C}$ kaum übersteigen.

Der Ofen wurde durch Quarzfenster verschlossen, die eine Bohrung zur Durchführung des Quarzrohres hatten; auf das letztere aufgeschobene Glimmerscheiben dichteten die Durchführung ab.

Die Optik vor den Fenstern wurde durch Kühlküvetten gegen die Wärmestrahlung geschützt und darüber hinaus durch einen Luftstrom gekühlt.

Als Lichtquelle diente eine 1000-W-Glühlampe, die mit einer Überspannung von $10 \%$ betrieben wurde. Sie beleuchtete eine Mattscheibe, von der durch eine Blende eine Kreisfläche ausgeblendet wurde.

Das Spektralphotometer wurde mit ein für allemal fest eingestelltem Kollimator- und Okularspalt verwendet. Die spektrale Breite des in die Photometerfelder gelangenden Lichtes betrug bei

$$
\begin{array}{ll}
4000 \AA & 11 \AA \\
5000 \AA & 27 \AA \\
6000 \AA & 57 \AA .
\end{array}
$$

Die Eichung der Trommel auf Wellenlängen erfolgte mit $\mathrm{He}, \mathrm{Hg}, \mathrm{H}$ und $\mathrm{Ne}$.

Die Absorptionsrohre wurden in der Weise gefüllt, daß die (bei kleinen Mengen auf der Mikrowaage abgewogene) S-Menge im Hochvakuum in das ausgeglühte Rohr hineindestilliert wurde; sodann wurde abgeschmolzen und das oben erwähnte Rohr angesetzt.

Zur Bestimmung der Extinktionskoeffizienten wurde zuerst bei Zimmertemperatur der Winkel $\alpha_{0}$ gemessen, und zwar, da er etwas mit der Wellenlänge variierte, durch das ganze Spektrum. Darauf wurde der Ofen angeheizt und nach Einstellung der jeweiligen Temperatur der Winkel $\alpha$ in Abständen von $100 \AA$ bestimmt, wobei mindestens 10 Einstellungen (in jedem Quadranten) vorgenommen wurden. Auf diese Weise wurden die Extinktionskoeffizienten bis etwa $900^{\circ} \mathrm{C}$ gemessen. Zur Kontrolle, ob die Justierung unverändert geblieben war und sich die Quarzscheiben bei den hohen Temperaturen nicht getrübt hatten, wurde jedesmal bei der höchsten Temperatur festgestellt, ob der Winkel $\alpha$ für die längsten Wellen von $7000 \AA$, wo dann keine merkliche Absorption mehr statt hat, mit dem anfangs gemessenen Winkel $\alpha_{0}$ identisch war.

Aus den Meßdaten wurden die Extinktionskoeffizienten $\varepsilon=1 / d \log I_{0} / I$ berechnet.

Zur Prüfung der Apparatur wurde bei einer Konzentration von $88 \mathrm{mg} / l$ und $490^{\circ} \mathrm{C}$ für eine Reihe von Wellenlängen der Extinktionskoeffizient mit Rohren

\begin{tabular}{|c|c|c|c|}
\hline Schichtdicke: & 48 & 24 & $6 \mathrm{~cm}$ \\
\hline Wellenlänge: & \multicolumn{3}{|c|}{$\varepsilon \cdot 10^{3}$} \\
\hline 4600 & 42,9 & 43,9 & 44,0 \\
\hline 4800 & 29,5 & 30,0 & 29,2 \\
\hline 5000 & 28,4 & 28,1 & 28,7 \\
\hline 5200 & 26,4 & 26,0 & 27,0 \\
\hline 5400 & 18,7 & 19,0 & 19,4 \\
\hline 5600 & 12,5 & 13,0 & 12,0 \\
\hline 5800 & 8,0 & 8,0 & 7,0 \\
\hline 6000 & 3,5 & 4,7 & \\
\hline
\end{tabular}
verschiedener Länge bestimmt. Eine Auswahl der Werte gibt Tab. 1 .

Tab. 1. Extinktionskoeffizienten für verschiedene Wellenlängen.

Ein systematischer Gang mit der Rohrlänge ist nicht zu erkennen.

Es wurden sodann 4 Meßreihen durchgeführt mit den Konzentrationen 43,5, 88, 261 und $1060 \mathrm{mg} / \mathrm{l}$. In jeder Reihe wurden die für die betreffenden Extinktionen passendsten Rohrlängen verwendet. In den Wellenlängenbereichen, in denen mit Rohren verschiedener Länge gemessen wurde, lagen die Abweichungen in dem gleichen Rahmen wie in Tab. 1; in Tab. 2 sind bei der Zusammenstellung der Ergebnisse die Mittelwerte aufgeführt.

\section{Ergebnisse und Diskussion}

In Abb. 1 und 2 sind für die kleinste und die größte Gesamtkonzentration die durch diese dividierten Extinktionswerte in Abhängigkeit von der Wellenlänge für die verschiedenen Temperaturen aufgetragen.

Die Extinktion nimmt vom violetten Ende her zunächst ab bis ca. $4700 \AA$, bei tiefen und hohen Temperaturen auch weiter bis zum völligen Verschwinden bei langen Wellen; bei einer mittleren Temperatur dagegen nimmt die Extinktion wieder bis zu einem sehr flachen Maximum bei $5100 \AA \mathrm{zu}$; danach nimmt sie auch hier bis Null bei langen 
Wellen ab. Für alle Wellenlängen gilt, daß mit wachsender Temperatur die Extinktion bis zu einem Maximum zu- und dann wieder abnimmt. Das muß dem Auftreten intermediärer Molekülarten $\left(S_{6}, S_{4}\right.$ oder beiden) zugeschrieben werden. Qualitativ wird dies durch den Vergleich von Abb. 1 und 2 bestätigt: bei der größeren Konzentration liegt die Temperatur des Maximums wesentlich höher.

Während ein Temperaturmaximum bei allen Wellenlängen auftritt, besteht jedoch ein Unterschied zwischen dem Verhalten des kurzwelligen Astes (bis ca. $4700 \AA$ ) und der anschließenden kontinuierlichen Absorption beiderseits 5100 A. Im kurzwelligen Teil ist auch bei tiefen und hohen Temperaturen eine merkliche Absorption vorhanden. Es überlagern sich dort offenbar mehrere Anteile. Bei hohen Temperaturen bleibt wenigstens bei der kleinsten Gesamtkonzentration nur der Anteil des $\mathrm{S}_{2}$ übrig. Bei tiefen Temperaturen könnte man zunächst an einen Beitrag von $\mathrm{S}_{8}$ denken. Da aber die Absorption des $S_{8}$ weit im UV liegt, ist nicht sehr wahrscheinlich, daß sie sich noch ins Sichtbare erstreckt. Auch sind Lösungen von $\mathrm{S}_{\mathrm{rh}}$ praktisch

\begin{tabular}{|c|c|c|c|c|c|c|c|c|c|c|}
\hline$t$ & $288^{\circ} \mathrm{C}$ & $359^{\circ} \mathrm{C}$ & $440^{\circ} \mathrm{C}$ & $490^{\circ} \mathrm{C}$ & $565^{\circ} \mathrm{C}$ & $640^{\circ} \mathrm{C}$ & $690^{\circ} \mathrm{C}$ & $790^{\circ} \mathrm{C}$ & $890^{\circ} \mathrm{C}$ & $990^{\circ} \mathrm{C}$ \\
\hline \multicolumn{11}{|c|}{ A. Gesamtkonzentration: $43,5 \mathrm{mg} / l$} \\
\hline 4400 & 5,9 & 12,4 & 27,1 & 43,8 & 56,2 & 42,3 & 33,8 & 21,0 & 15,1 & 8,7 \\
\hline 4500 & 4,5 & 8,8 & 18,6 & 27,2 & 34,3 & 26,1 & 21,5 & 14,7 & 9,3 & 6,4 \\
\hline 4600 & 3,2 & 7,1 & 14,2 & 21,1 & 24,4 & 18,3 & 14,9 & 9,9 & 7,1 & 4,0 \\
\hline 4700 & 2,2 & 4,9 & 10,3 & 16,5 & 16,9 & 11,1 & 8,5 & 5,3 & 4,0 & 2,3 \\
\hline 4800 & 1,9 & 3,6 & 9,5 & 14,3 & 14,1 & 7,5 & 6,2 & 3,0 & 2,3 & 1,0 \\
\hline 4900 & 1,3 & 3,9 & 9,5 & 14,4 & 14,1 & 6,3 & 5,0 & 2,5 & 1,1 & 0,8 \\
\hline 5000 & 1,2 & 3,6 & 9,5 & 15,5 & 14,2 & 6,2 & 4,6 & 2,2 & 0,9 & - \\
\hline 5100 & 1,0 & 3,4 & 10,1 & 15,5 & 14,4 & 5,8 & 3,7 & 1,6 & 0,7 & - \\
\hline 5200 & - & 3,2 & 10,2 & 14,8 & 14,4 & 5,6 & 3,5 & 1,1 & - & - \\
\hline 5300 & 0,6 & 3,2 & 8,6 & 14,2 & 13,0 & - & 3,3 & 1,0 & - & - \\
\hline 5400 & - & - & - & - & 11,5 & 5,2 & 2,7 & 0,9 & - & - \\
\hline 5500 & - & 2,2 & 6,2 & 9,7 & 9,2 & - & 2,2 & - & - & - \\
\hline 5600 & - & - & - & - & 8,5 & 3,9 & 1,8 & 0,8 & - & - \\
\hline 5700 & 0,2 & 0,9 & 4,1 & 6,8 & - & - & - & - & 一 & - \\
\hline 5800 & - & - & - & - & 5,2 & 2,3 & 1,1 & 0,5 & - & $\ldots$ \\
\hline 6000 & - & 0,4 & 1,8 & 4,0 & 4,0 & 1,4 & 0,5 & 0,3 & - & - \\
\hline 6300 & - & - & - & - & 1,9 & 0,8 & - & - & - & - \\
\hline 6500 & - & - & 0,8 & 0,8 & - & - & - & - & - & - \\
\hline 6600 & - & -. & - & - & 1,8 & - & - & - & - & - \\
\hline 6700 & - & - & - & 0,4 & - & - & - & - & - & - \\
\hline \multicolumn{11}{|c|}{ B. Gesamtkonzentration $88 \mathrm{mg} / \mathrm{l}$} \\
\hline 4400 & 10,4 & 20,4 & 46,5 & 95,0 & 135 & 124 & 104 & 67,5 & 43,0 & - \\
\hline 4500 & 7,2 & 14,5 & 33,1 & 66,5 & 96,0 & 85,5 & 73,5 & 49,0 & 32,3 & 26,0 \\
\hline $46 C 0$ & 5,1 & 11,2 & 25,1 & 44,0 & 68,0 & 57,0 & 48,6 & 32,5 & 22,0 & 17,0 \\
\hline 4700 & 3,9 & 8,5 & 20,4 & 31,8 & 47,2 & 36,3 & 29,1 & 20,2 & 13,7 & 11,0 \\
\hline 4800 & 2,8 & 6,7 & 18,2 & 28,8 & 39,5 & 26,9 & 20,6 & 13,0 & 9,5 & 7,0 \\
\hline 4900 & 2,4 & 5,8 & 17,4 & 28,6 & 39,3 & 24,5 & 17,1 & 10,6 & 7,0 & 6,0 \\
\hline 5000 & 1,9 & 5,0 & 17,1 & 28,9 & 39,3 & 23,4 & 16,0 & 9,0 & 6,8 & 5,3 \\
\hline 5100 & 1,5 & 4,7 & 17,0 & 29,0 & 39,1 & 22,7 & 14,6 & 8,0 & 6,0 & 4,5 \\
\hline 5200 & 1,2 & 3,5 & 15,7 & 26,5 & 38,0 & 22,0 & 14,6 & 7,2 & 5,0 & 3,5 \\
\hline 5300 & - & 3,0 & 14,0 & 23,7 & 34,6 & 20,3 & 13,5 & 6,3 & 4,3 & 3,0 \\
\hline 5400 & 0,8 & 2,6 & 11,8 & 20,0 & 30,3 & 18,3 & 12,3 & 5,5 & 4,0 & 2,7 \\
\hline 5500 & - & - & 9,6 & 16,8 & 25,1 & 15,5 & 10,7 & 4,9 & 3,0 & 2,0 \\
\hline 5600 & 0,6 & 1,5 & 7,9 & 12,9 & 20,4 & 12,9 & 8,6 & 4,0 & - & 1,9 \\
\hline 5700 & - & - & - & 9,9 & 16,3 & 10,6 & 7,1 & 3,0 & 2,0 & 1,8 \\
\hline 5800 & 0,4 & 0,8 & 4,6 & 8,0 & 13,0 & 8,6 & - & 2,5 & - & - \\
\hline 5900 & - & - & - & - & - & 6,7 & - & 2,0 & - & - \\
\hline 6000 & 0,3 & 0,3 & 3,0 & 4,7 & 8,2 & 5,5 & 2,6 & 1,8 & 0,5 & 0,5 \\
\hline 6200 & 0,2 & 0,2 & 2,0 & 2,8 & 5,0 & 4,5 & - & 1,0 & - & - \\
\hline 6400 & 0,1 & - & 0,7 & 1,5 & - & - & - & - & - & - \\
\hline 6500 & - & - & 0,5 & 1,0 & 1,8 & 1,3 & 0,4 & - & - & - \\
\hline
\end{tabular}

Tab. 2. Extinktionskoeffizienten $\varepsilon \cdot 10^{3}$ für verschiedene Temperaturen und verschiedene Wellenlängen. 


\begin{tabular}{|c|c|c|c|c|c|c|c|c|}
\hline$t$ & $359^{\circ} \mathrm{C}$ & $440^{\circ} \mathrm{C}$ & $490^{\circ} \mathrm{C}$ & $565^{\circ} \mathrm{C}$ & $640^{\circ} \mathrm{C}$ & $690^{\circ} \mathrm{C}$ & $790^{\circ} \mathrm{C}$ & $890^{\circ} \mathrm{C}$ \\
\hline \multicolumn{9}{|c|}{ C. Gesamtkonzentration: $261 \mathrm{mg} / \mathrm{l}$} \\
\hline $\begin{array}{l}4400 \\
4500 \\
4600 \\
4700 \\
4800 \\
4900 \\
5000 \\
5100 \\
5200 \\
5300 \\
5400 \\
5500 \\
5600 \\
5700 \\
5900 \\
6000 \\
6200 \\
6300 \\
6400 \\
6500 \\
6600 \\
6700 \\
6800\end{array}$ & $\begin{array}{r}56,5 \\
40,1 \\
31,8 \\
23,4 \\
19,4 \\
15,6 \\
13,4 \\
11,4 \\
9,5 \\
6,9 \\
- \\
- \\
3,0 \\
1,7 \\
- \\
- \\
- \\
- \\
- \\
-\end{array}$ & $\begin{array}{r}115 \\
84 \\
65,9 \\
53,8 \\
46,8 \\
42,6 \\
39,6 \\
37,4 \\
33,9 \\
28,8 \\
- \\
15,3 \\
- \\
7,8 \\
-5,0 \\
4,3 \\
-3,2 \\
-2,8 \\
-\end{array}$ & $\begin{array}{r}183 \\
132 \\
104 \\
81,0 \\
74,0 \\
71,4 \\
70,1 \\
67,5 \\
64,2 \\
57,5 \\
49,1 \\
39,3 \\
31,5 \\
- \\
16,3 \\
13,2 \\
9,2 \\
-6,3 \\
4,4 \\
3,1 \\
1,8\end{array}$ & $\begin{array}{r}360 \\
234 \\
184 \\
152 \\
135 \\
135 \\
136 \\
133 \\
127 \\
115 \\
99,0 \\
85,0 \\
70,4 \\
- \\
38,6 \\
32,6 \\
23,6 \\
-16,3 \\
12,2 \\
-7,0\end{array}$ & $\begin{array}{r}421 \\
304 \\
227 \\
161 \\
138 \\
134 \\
131 \\
132 \\
127 \\
116 \\
103 \\
88,1 \\
75,0 \\
-\overline{4,2} \\
38,4 \\
28,5 \\
-1,4 \\
15,9 \\
11,4\end{array}$ & $\begin{array}{r}393 \\
282 \\
208 \\
142 \\
115 \\
104 \\
99,0 \\
98,0 \\
95,0 \\
89,0 \\
80,0 \\
69,5 \\
- \\
- \\
- \\
- \\
- \\
- \\
- \\
-\end{array}$ & $\begin{array}{r}280 \\
213 \\
139 \\
97,1 \\
70,0 \\
54,7 \\
45,4 \\
40,3 \\
36,6 \\
33,7 \\
- \\
22,6 \\
- \\
\overline{13,0} \\
10,6 \\
- \\
\overline{7,4} \\
\overline{4,4} \\
\end{array}$ & $\begin{array}{r}190 \\
136 \\
110 \\
79,9 \\
53,5 \\
38,8 \\
29,4 \\
23,6 \\
20,0 \\
17,2 \\
- \\
12,0 \\
-8,7 \\
-6,4 \\
4,0 \\
- \\
3,8 \\
05 \\
\end{array}$ \\
\hline \multicolumn{9}{|c|}{ D. Gesamtkonzentration: $1060 \mathrm{mg} / \mathrm{l}$} \\
\hline $\begin{array}{l}4400 \\
4500 \\
4600 \\
4700 \\
4800 \\
4900 \\
5000 \\
5100 \\
5200 \\
5300 \\
5400 \\
5500 \\
5600 \\
5700 \\
5800 \\
6000 \\
6200 \\
6500 \\
6700\end{array}$ & $\begin{array}{r}205 \\
143 \\
103 \\
75,3 \\
61,9 \\
49,5 \\
41,9 \\
33,4 \\
20,0 \\
- \\
7,3 \\
- \\
- \\
1,7 \\
- \\
- \\
-\end{array}$ & $\begin{array}{r}396 \\
284 \\
223 \\
172 \\
143 \\
127 \\
116 \\
101 \\
89,7 \\
75,6 \\
48,0 \\
29,0 \\
16 \\
- \\
7 \\
6\end{array}$ & $\begin{array}{c}580 \\
412 \\
331 \\
269 \\
226 \\
213 \\
202 \\
185 \\
170 \\
149 \\
128 \\
\overline{78,7} \\
\overline{45} \\
30 \\
\overline{17} \\
8\end{array}$ & $\begin{array}{r}755 \\
7530 \\
503 \\
454 \\
444 \\
441 \\
434 \\
411 \\
371 \\
310 \\
269 \\
218 \\
149 \\
122 \\
82 \\
41\end{array}$ & $\begin{array}{r}12 \overline{15} \\
950 \\
715 \\
684 \\
698 \\
697 \\
696 \\
675 \\
624 \\
553 \\
482 \\
405 \\
- \\
284 \\
207 \\
157 \\
96 \\
78\end{array}$ & $\begin{array}{r}\overline{1425} \\
1100 \\
910 \\
815 \\
744 \\
744 \\
738 \\
721 \\
667 \\
601 \\
\overline{450} \\
-\overline{304} \\
246 \\
188 \\
124 \\
91\end{array}$ & $\begin{array}{r}-\overline{1330} \\
1000 \\
805 \\
617 \\
538 \\
492 \\
466 \\
443 \\
414 \\
375 \\
336 \\
290 \\
- \\
217 \\
175 \\
133 \\
87 \\
74\end{array}$ & $\begin{array}{r}-\overline{60} \\
775 \\
611 \\
425 \\
352 \\
298 \\
262 \\
239 \\
-\overline{190} \\
- \\
145 \\
- \\
-- \\
- \\
48 \\
-\end{array}$ \\
\hline
\end{tabular}

Fortsetzung der Tab. 2.

farblos. Es wäre dagegen möglich, daß die im kurzwelligen Teil bei tiefen Temperaturen zu beobachtende Absorption von $\mathrm{S}_{6}$ herrührt, das hier bereits in merklicher Konzentration vorhanden ist. Damit würde die Tatsache übereinstimmen, daß nach noch unveröffentlichten Versuchen von Poulsen-Nautrup Lösungen von Engelschem Schwefel, die in Bestätigung von Messungen von Aten ${ }^{5}$, den Schwefel in Form von $\mathrm{S}_{6}$ enthalten, eine deut-

5 A. H.W. Aten, Z. f. physik. Chem. 88, 321 [1914]. liche, mit wachsender Wellenlänge abnehmende Extinktion zeigen; diese Lösungen sind auch stark gelb gefärbt. Jedoch ist die molare Extinktion der Lösungen sehr viel kleiner als diejenige, welche sich ergibt, wenn man die Absorption im Gas ausschließlich dem $\mathrm{S}_{6}$ zuschreibt und auf Grund der $\mathrm{Zu}$ sammensetzung die molare $\mathrm{S}_{6}$-Extinktion berechnet. Danach scheint die $\mathrm{S}_{6}$-Absorption nur wenig zur Extinktion in diesem Gebiet beizutragen, und sie muß daher wohl in der Hauptsache von $\mathrm{S}_{4}$ herrühren. 


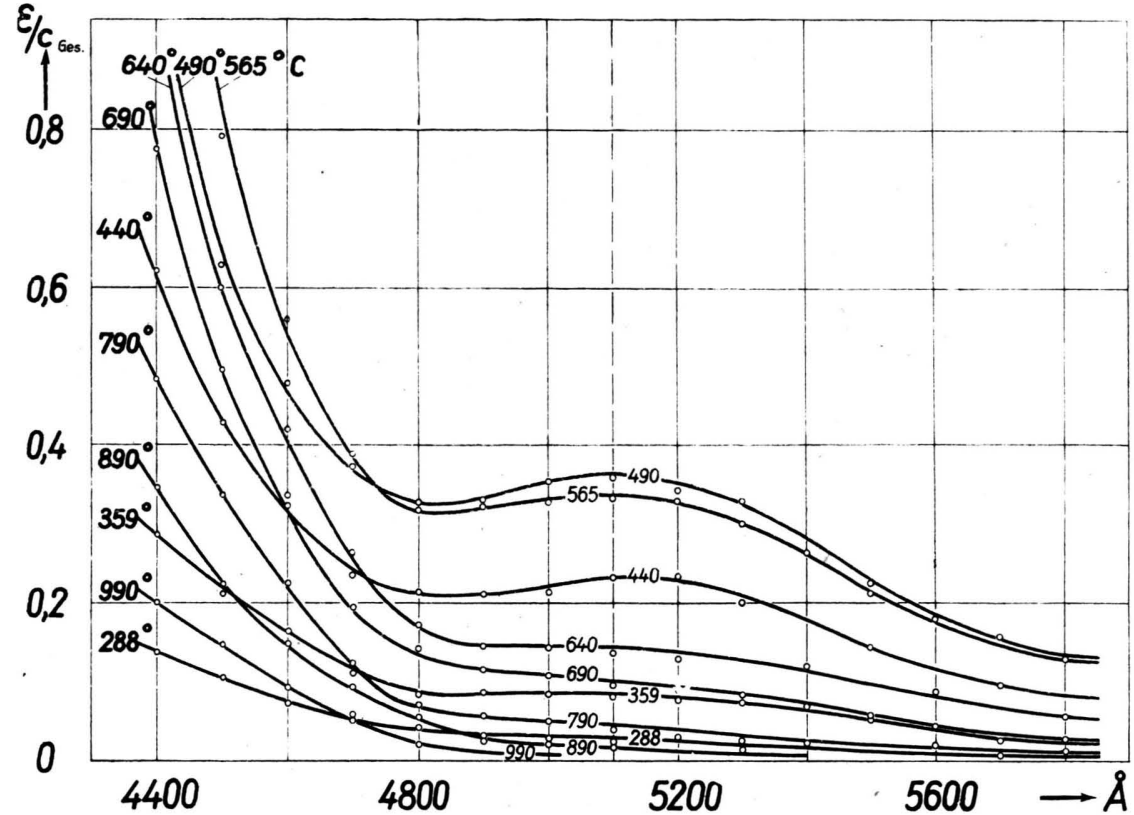

Abb. 1. Extinktionswerte $\varepsilon / c$ ges in Abhängigkeit von der Wellenlänge für die kleinste Gesamtkonzentration ( $\left.c_{\text {ges }}=43,5 \mathrm{mg} / \mathrm{l}\right)$.

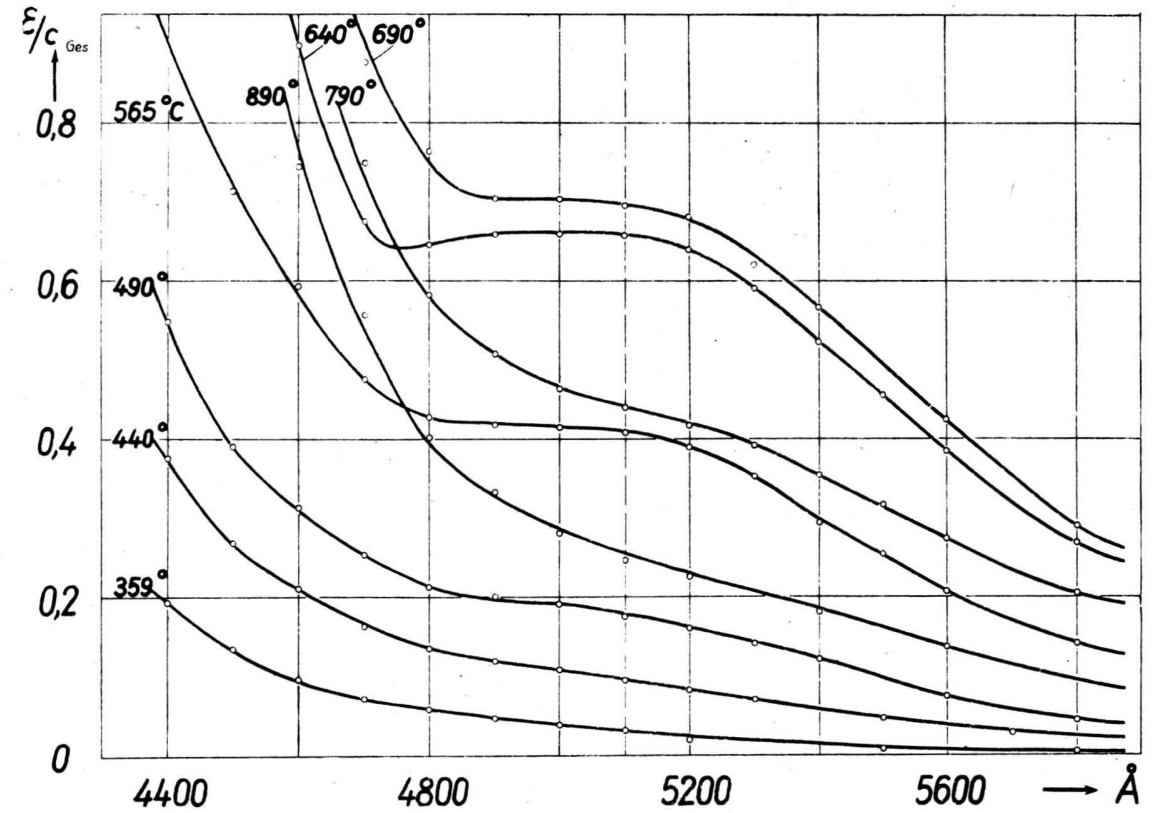

Abb. 2. Extinktionswerte $\varepsilon / c_{\text {ges }}$ in Abhängigkeit von der Wellenlänge für die größte Gesamtkonzentration $\left(c_{\mathrm{ges}}=1060 \mathrm{mg} / l\right)$.
Das gleiche gilt für die $S_{2^{-}}$ Absorption. Daß bei der großen Konzentration $(D)$ im Gebiet hoher Temperaturen die Absorption noch nicht auf Null zurückgegangen ist, hat seinen Grund darin, daß hier die Konzentration der für die Bande um $5100 \AA$ verantwortlichen Molekülart selbst bei den höchsten Beobachtungs temperaturen noch nicht weit genug zurückgegangen ist.

Bei $5100 \AA$ scheinen daher dieVerhältnisse wesentlich einfacher zu liegen, und es ist nun zu entscheiden, ob diese Bande von $\mathrm{S}_{6}$ oder, wie nach dem Gesagten wahrscheinlicher ist, von $\mathrm{S}_{4}$ herrührt. Das Folgende bezieht sich daher auf dieWellenlänge $5100 \AA$.

Wenn die Konzentration der für die Extinktion verantwortlichen Molekülart $\mathrm{S}_{\mathrm{i}}$ und $c_{\mathrm{i}}$ (in Mol/ll) und ihr molarerExtinktionskoeffizient mit $k_{\mathrm{i}}$ bezeichnet werden, so ist nach dem Beerschen Gesetz

$$
E=k_{\mathrm{i}} c_{\mathrm{i}} d ; \varepsilon=k_{\mathrm{i}} c_{\mathrm{i}} .
$$

Dabei werde, was vielleicht nicht streng zutrifft, $k_{\mathrm{i}}$ als von der Temperatur unabhängig angenommen.

Die Konzentrationen der im Dampf vorhandenen Molekülarten lassen sich aus den Gleichgewichten (1) bis (3) berechnen.

Bei 5100 Å dagegen ist bei der kleinsten Konzentration sowohl bei den tiefen wie bei den hohen Temperaturen keine praktisch wesentlich ins Gewicht fallende Extinktion vorhanden. Der Ausläufer des kurzwelligen Astes, soweit, er von $S_{6}$ herrührt, ist also offenbar bei $5100 \AA \AA$ schon so schwach, daß er nicht merklich zur Absorption beiträgt.
Es ist, wenn $v$ die durchschnittliche Zahl der A tome im Molekül bedeutet:

$$
v P=\frac{G}{A V} R T
$$

( $G=$ Gewicht des Schwefels, $V=$ Volumen des Absorptionsrohrs). 
Ferner ist ${ }^{1}$

$$
\begin{gathered}
2 p_{2}+4 p_{4}+6 p_{6}+8 p_{8}=v P, \\
2 p_{2}+4 \frac{p_{2}{ }^{2}}{K_{4}}+6 \frac{p_{2}{ }^{3}}{K_{6}}+8 \frac{p_{2}{ }^{4}}{K_{8}}=v P,
\end{gathered}
$$

wenn mit $p_{\mathrm{i}}$ der Partialdruck von $\mathrm{S}_{\mathrm{i}}$ bezeichnet wird.

$\nu P$ ist nach (4) durch die Einwaage gegeben. Aus (5) lassen sich die einzelnen Partialdrucke berechnen, indem man $P_{2}$ solange variiert, bis die Summe der linken Seite gleich $v P$ wird. Aus den Partialdrucken ergeben sich dann die Konzentrationen.

Das Ergebnis wird für die Konzentration $261 \mathrm{mg} / l$ durch Abb. 3 veranschaulicht. Als Ordinaten sind die Konzentrationen der einzelnen Molekülarten aufgetragen, für $\mathrm{S}_{6}$ auch die nach den alten Messungen von Preuner und Schupp berechneten. Ferner ist die Extinktionskurve aufgetragen, wobei der Maßstab so gewählt ist, daß für die Temperatur des

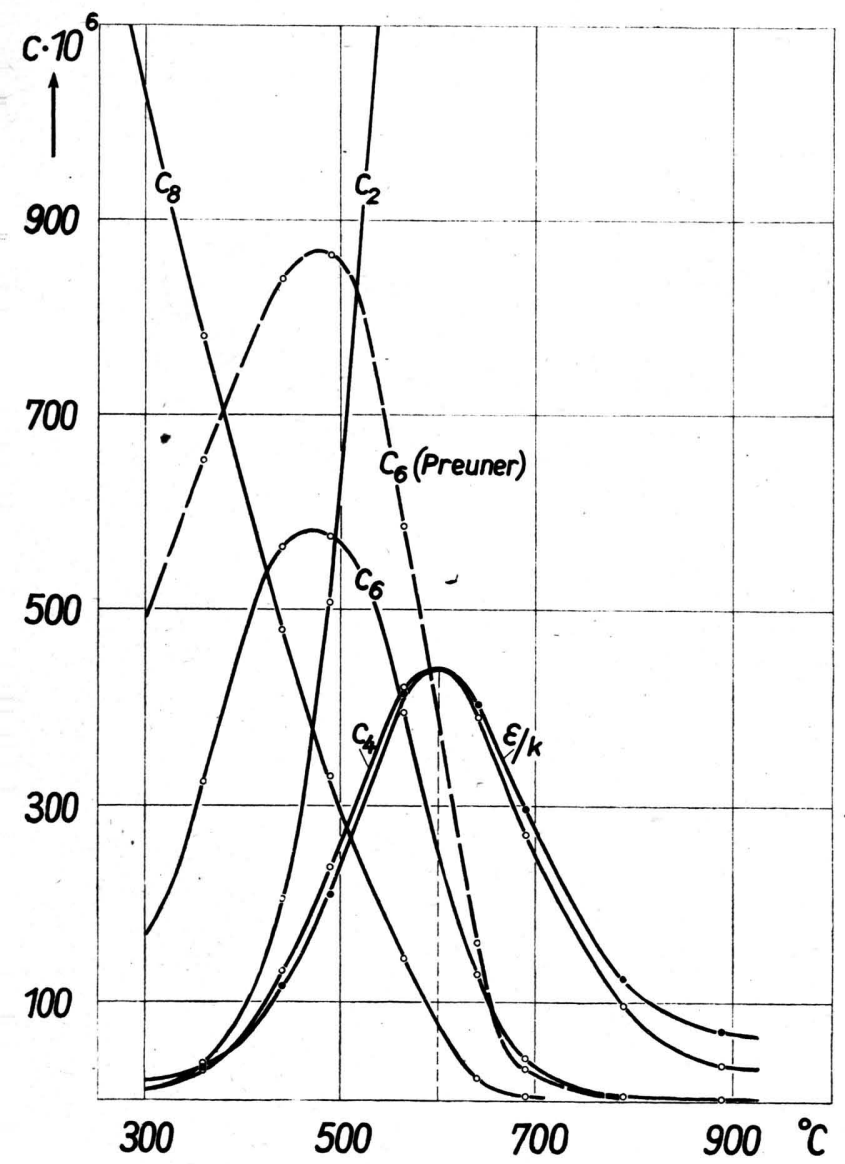

Abb. 3. Vergleich der molaren Einzelkonzentrationen mit dem Verlauf von $\varepsilon / k$ für $c_{\mathrm{ges}}=261 \mathrm{mg} / \mathrm{l}$.

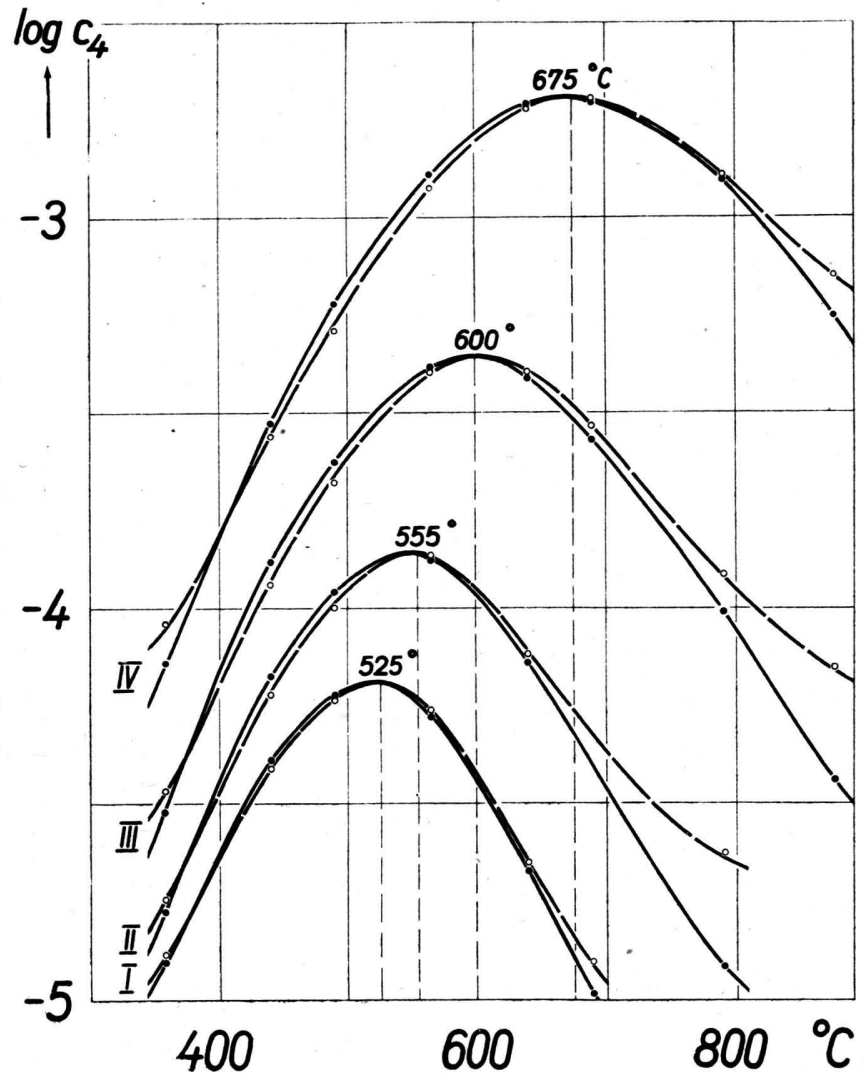

Abb. 4. $\log c_{4}$ und $\log \varepsilon / k$ in Abhängigkeit von der Temperatur. $\bullet-\bullet \log c_{4} ; \circ--\circ \log \varepsilon / k$, Kurve I $: c_{\text {ges }}=$ $43,5 \mathrm{mg} / l$, Kurve II: $c_{\text {ges }}=88 \mathrm{mg} / l$, Kurve III: $c_{\text {ges }}=261 \mathrm{mg} / l$, Kurve IV $: c_{\text {ges }}=1060 \mathrm{mg} / l$.

Extinktionsmaximums die Ordinate gleich $c_{4 \max }$ ist, d. h. es ist

$$
\varepsilon_{\max } / k=c_{4 \max } \cdot
$$

Wenn die Extinktion von $\mathrm{S}_{4}$ herrührt, hat $k$, das zunächst nur durch den Normierungsfaktor definiert ist, die Bedeutung des molaren Extinktionskoeffizienten von $\mathrm{S}_{4}$, ist also als $k_{4}$ zu bezeichnen.

Man erkennt, daß nur zwischen den $c_{4}$-Werten und der Extinktion Parallelismus besteht: die Temperatur des Maximums ist innerhalb der Fehlergrenzen die gleiche und auch der Verlauf der Kurve ist analog, während für $\mathrm{S}_{6}$ das Maximum der Konzentration bei einer um ca. $150^{\circ} \mathrm{C}$ niedrigeren Temperatur liegt als das Extinktionsmaximum. Ganz entsprechend sind die Bilder für die anderen Konzentrationen. In Abb. 4 sind die $\varepsilon / k$ und $c_{4}$ für alle 4 Versuchsreihen logarithmisch gegen die Temperatur aufgetragen. Es zeigt sich für alle vier Konzentrationen eine gleich gute Übereinstimmung zwischen den beiden Kurven, obgleich die Kon- 
zentrationen im Verhältnis $1: 24,2$ variiert sind und die Temperatur des Maximums mit wachsender Gesamtkonzentration von etwa 525 auf $675^{\circ} \mathrm{C}$ ansteigt. Außerdem sind die $k$-Werte der Maxima für die 4 Versuchsreihen nahezu gleich (Tab. 3).

\begin{tabular}{|c|c|c|}
\hline $\begin{array}{c}\text { Gesamt- } \\
\text { konzentration } \\
\mathrm{mg} / l\end{array}$ & $\begin{array}{c}\text { Temperatur } \\
\text { des Maximums } \\
{ }^{\mathbf{0}} \mathbf{C}\end{array}$ & $k_{\mathbf{4}}$ \\
\hline 43,5 & 525 & 260 \\
88 & 555 & 288 \\
261 & 600 & 325 \\
1060 & 675 & 363 \\
\hline
\end{tabular}

Tab. 3. Molarer Extinktionskoeffizient des $\mathbf{S}_{4}$ für $\lambda=5100$ Å bei Temperaturen maximaler Extinktion und verschiedenen Gesamtkonzentrationen.

In Tab. 4 sind noch für die 4 Versuchsreihen die Werte $k_{4}=\varepsilon / c_{4}$ bei verschiedenen nicht dem Maximum entsprechenden Temperaturen zusammengestellt, ferner sind die Werte angeführt, die sich ergeben, wenn man die Extinktion dem $\mathrm{S}_{6}$-Molekül zuzuschreiben versucht $\left(k_{6}=\varepsilon / c_{6}\right.$ bzw. $\left.k_{6}=\varepsilon / c_{6 \text { Preuner }}\right)$.
Es zeigt sich, daß nur die Annahme; daß die Extinktion von $\mathrm{S}_{4}$ herrührt, bei gegebener Temperatur einen von der Gesamtkonzentration unabhängigen Wert von $k$ liefert, während $k_{6}$ und $k_{6} \operatorname{Pr}$ eine außerordentlich starke Konzentrationsabhängigkeit zeigen. Auch die Temperatur hat auf $k_{4}$ einen sehr geringen Einfluß im Gegensatz zu $k_{6}$ und $k_{6}$ Pr. Dabei variieren die $c_{4}$-Werte fast wie $1: 200$. Es ist also eindeutig bewiesen, daß die Annahme von Rosen und Neven ${ }^{3}$, wonach die $\mathrm{Ab}$ sorption im Sichtbaren der Ausläufer eines sich weit ins sichtbare Gebiet erstreckenden $\mathrm{S}_{6}$-Kontinuums sein sollte, zum mindesten für die kontinuierliche Bande um $5100 \AA$ nicht zutreffen kann. Die $\mathrm{S}_{6}$ Absorption kann vielmehr bei $5100 \AA$ keine wesentliche Rolle spielen, wie schon aus den relativ kleinen Extinktionen hervorgeht, die bei allen 4 Versuchsreihen für die Temperaturen gefunden werden, bei denen $c_{6}$ seinen Maximalwert besitzt. Vielmehr steht fest, daß die kontinuierliche Absorption mit dem Maximum bei $5100 \AA$ von $\mathrm{S}_{4}$ herrührt.

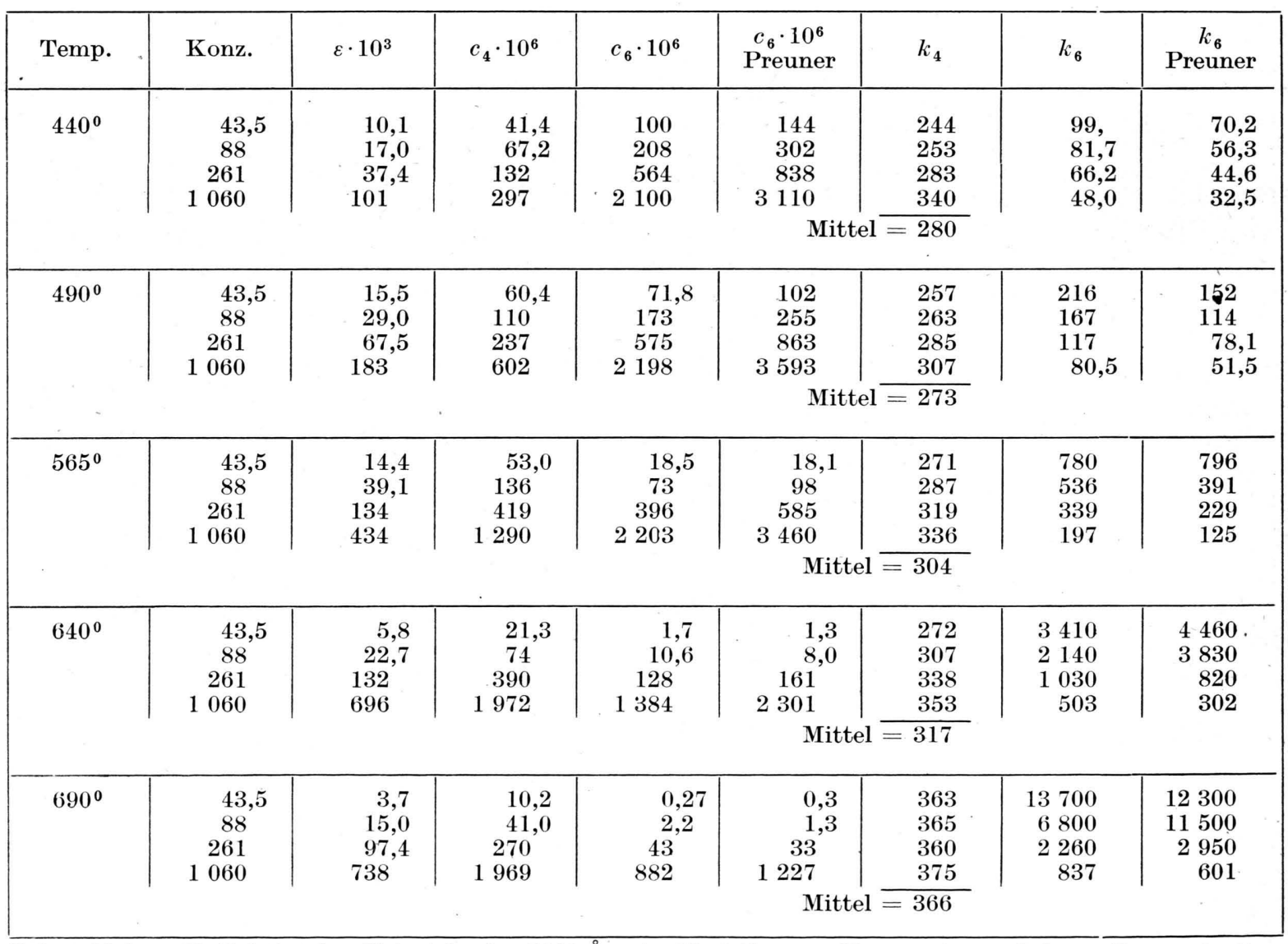

Tab. 4. Molarer Extinktionskoeffizient für $\lambda=5100 \AA$ unter Variation von Temperatur und Gesamtkonzentration bei verschiedenen Annahmen über die Art des absorbierenden Moleküls. 
Daß auch bei kleineren Wellenlängen $S_{6}$ nur sehr wenig zur Extinktion beiträgt, wie oben schon wahrscheinlich gemacht war, geht aus der Tatsache hervor, daß Kurven, die die Extinktion in Abhängigkeit von der Temperatur darstellen, bei allen, auch bei den kurzen Wellenlängen, im Temperaturgebiet des Maximums der $\mathrm{S}_{6}$-Konzentration einen völlig glatten Verlauf zeigen; es ist also von einer Überlagerung durch eine $\mathrm{S}_{6}$-Absorption nichts $\mathrm{zu}$ erkennen. In untergeordnetem Maße kann ein Ausläufer der kurzwelligeren $\mathrm{S}_{2}$-Absorption zur Extinktion beitragen, wovon vielleicht das merkliche Differieren der $c_{4^{-}}$und $\varepsilon / k$-Kurven im Gebiet hoher Temperaturen bei kleinen $c_{4}$-Werten herrührt. Die gute Übereinstimmung der aus den Absorptionsmessungen gezogenen Schlüsse mit den Ergebnissen der Dampfdichtebestimmungen betrachten wir umgekehrt als starke Stütze für die Richtigkeit der abgeleiteten Gleichungen für die Dissoziationskonstanten.

Es ist von Interesse, darauf hinzuweisen, daß schon D'Or ${ }^{4}$ betont hat, daß die (qualitativen) Anhaltspunkte, die sich aus Spektralaufnahmen gewinnen ließen, seiner Ansicht nach die Annahme einer weiteren intermediären Molekülart neben $\mathrm{S}_{6}$ erforderten.
Wie bereits erwähnt, liegen im kurzwelligen Teil des Spektrums $(\lambda<4700 \AA)$ die Verhältnisse insofern verwickelter, als hier offenbar bei tieferen Temperaturen $\mathrm{S}_{6}$ merklich zur Absorption beiträgt, während bei hohen Temperaturen, wo nur noch $\mathrm{S}_{2}$ übrig bleibt, dieses eine meßbare Absorption zeigt. Im mittleren Gebiet ist auch bei diesen Wellenlängen die Absorption zum überwiegenden Teile dem $\mathrm{S}_{4}$ zuzuschreiben, wie sich schon daraus ergibt, daß die Temperatur des Maximums bei allen Wellenlängen dieses ganzen Gebietes nicht wesentlich verschieden ist von dem derWellenlänge $5100 \AA$, es liegt nur etwa $30^{\circ}$ höher als dort.

Im kurzwelligen Teil ist übrigens auf Spektralaufnahmen eine sehr diffuse Bandenstruktur zu sehen, die nach längeren Wellen immer undeutlicher wird und ab $4600 \AA$ kaum mehr zu erkennen ist. Photometrierung von bei steigender Temperatur aufgenommenen Spektren zeigte mit aller Deutlichkeit, daß diese Banden bei tiefen und hohen Temperaturen verschwinden und ihre stärkste Intensität bei der Temperatur des $c_{4}$-Maximums haben. Daraus folgt), daß diese Banden, die Rosen und Neven dem $S_{2}$-Molekül zuzuordnen versucht hatten, zwangloser dem $\mathrm{S}_{4}$ zugeschrieben werden können.

\title{
Über die Intensitätsabhängigkeit der Quantenbilanz photochemischer Prozesse
}

\author{
Von Karl H. Hausser und Udo Wegner \\ Aus dem Max-Planck-Institut für medizinische Forschung, Heidelberg \\ Abteilung für physikalische.Therapie \\ (Z. Naturforschg. 7 a, 493-497 [1952]; eingegangen am 26. November 1951)
}

\begin{abstract}
Es wird im Anschluß an ein experimentelles Ergebnis untersucht, wieso bei einem photochemischen Vorgang, dessen Dosis-Effekt-Kurve den Charakter einer EintrefferKurve hat, eine Abhängigkeit der pro umgewandeltem Molekül absorbierten Quanten von der Intensität des eingestrahlten Lichtes auftreten kann. Dabei zeigt es sich, daß in bestimmten Fällen trotzVorliegens eines Zweitreffer-Vorganges eineEintreffer-Kurve vorgetäuscht werden kann und zwar dann, wenn der Zwischenzustand instabil und von kurzer Lebensdauer ist und wenn sein Absorptionsquerschnitt sich von demjenigen des Ausgangszustandes erheblich unterscheidet. Es werden der theoretische Verlauf der Umwandlungs-Kurven in einem solchen Fall berechnet und einige Kurvenscharen als Beispiele gegeben. Mit Hilfe der gewonnenen Formeln läßt sich bei Vorliegen von_mehreren bei verschiedenen Lichtintensitäten gemessenen Umwandlungs-Kurven die Halbwertzeit und der Absorptionsquerschnitt des experimentell nicht erfaßbaren instabilen Zwischenzustandes berechnen.
\end{abstract}

$\mathrm{E}$ iner von uns erhielt bei der experimentellen Untersuchung der Quantenbilanz bei der rot $\rightleftarrows$ gelb-Umwandlung von Triphenylformazan ${ }^{1}$

${ }^{1}$ K. H. Hausser, Z. Naturforschg. 5a, $41[1950$ |.

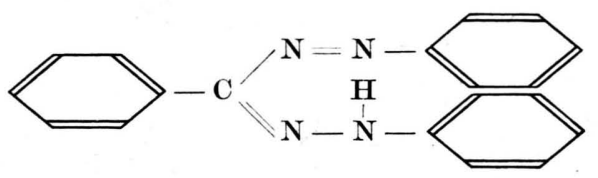

